\title{
Gutachter der Zeitschrift 2000/2001
}

An der Begutachtung von Manuskripten, die der Zeitschrift im Zeitraum zwischen dem 1. Juli 2000 und dem 30. Juni 2001 eingereicht wurden, haben die folgenden Kolleginnen und Kollegen mitgewirkt. Wir danken ihnen sehr herzlich für die Beteiligung an der Entstehung der Zeitschrift.

\author{
Alpheis, Hannes (Hamburg) \\ Bach, Maurizio (Bamberg) \\ Bamberg, Sebastian (Gießen) \\ Blomert, Reinhard (Berlin) \\ Bös, Mathias (Heidelberg) \\ Brettschneider, Frank (Stuttgart) \\ Brose, Hanns-Georg (Duisburg) \\ Brosziewski, Achim (St. Gallen) \\ Brüderl, Josef (Mannheim) \\ Bulmahn, Thomas (Berlin) \\ Erb, Rainer (Potsdam) \\ Fetchenhauer, Detlef (Bochum) \\ Fuchs-Heinritz, Werner (Hagen) \\ Gebauer, Gunter (Berlin) \\ Halfmann, Jost (Dresden) \\ Hammerich, Kurt (Aachen) \\ Häußermann, Hartmut (Berlin) \\ Heintz, Bettina (Mainz) \\ Huinink, Johannes (Rostock) \\ Jansen, Dorothea (Speyer) \\ Junge, Matthias (Chemnitz) \\ Kalter, Frank (Mannheim)
}

\author{
Kepplinger, Hans Mathias (Mainz) \\ Klein, Thomas (Heidelberg) \\ Kohl, Jürgen (Heidelberg) \\ Konietzka, Dirk (Rostock) \\ Krais, Beate (Darmstadt) \\ Kühl, Stefan (München) \\ Lindner-Braun, Christa (Köln) \\ Meulemann, Heiner (Köln) \\ Motel, Andreas (Berlin) \\ Müller-Benedict, Volker (Göttingen) \\ Neuendorff, Hartmut (Dortmund) \\ Preisendörfer, Peter (Rostock) \\ Roller, Edeltraud (Berlin) \\ Schmid, Michael (Augsburg) \\ Teckenberg, Wolfgang (Heidelberg) \\ Trommsdorff, Gisela (Konstanz) \\ Voß, Günter G. (Chemnitz) \\ Weede, Erich (Bonn) \\ Welzel, Christian (Berlin) \\ Werle, Raymund (Köln) \\ Windolf, Paul (Trier) \\ Wittenberg, Reinhard (Nürnberg)
}

